\title{
Effects of trust, structure, and processes on effectiveness in a military organization - exploring a moderated mediation model
}

\author{
Anne Lise Bjørnstad \\ Norwegian Defence Research Establishment (FFI) \\ Pål Ulleberg \\ University of Oslo
}

\section{Citation}

Bjørnstad, A. L. \& Ulleberg, P. (2021). Effects of trust, structure, and processes on effectiveness in a military organization: exploring a moderated mediation model. Leadership \& Organization Development Journal, Vol. 42 No. 4, pp. 564-579. https://doi.org/10.1108/LODJ-06-2020-0255

Publisher: Emerald Publishing Limited.

Copyright (C) 2021, Emerald Publishing Limited. This author accepted manuscript (AAM) is provided for your own personal use only. It may not be used for resale, reprinting, systematic distribution, emailing, or for any other commercial purpose without the permission of the publisher. 


\begin{abstract}
Authors' note
Anne Lise Bjørnstad, Department of Strategic Analyses and Joint Systems, Norwegian Defence Research Establishment (FFI), Norway; Pål Ulleberg, Department of Psychology, University of Oslo, Norway.

The authors would like to thank colleagues at the Norwegian Defence Research Establishment (FFI), Hilde Hafnor and Ann Kristin Elstad for their much valued part in the data collection. The authors thank also go out to all the military participants and the units that cooperated with the authors to make this data collection possible.

Correspondence should be addressed to Anne Lise Bjørnstad, Norwegian Defence Research Establishment (FFI), P. O. Box 25, 2027 Kjeller, Norway; e-mail: annelise.bjornstad@,ffi.no.
\end{abstract}




\begin{abstract}
Purpose - The purpose of this study is to better understand the consequences of trust between personnel at different hierarchical levels for organizational effectiveness. We aim to explore the direct effects and the interaction effects of trust with organizational structure and processes. The study focuses on military organizations and expands on models and research from
\end{abstract} this context.

Design/methodology/approach - Survey data were collected from a Norwegian military exercise organization at two different hierarchical levels. The hypothesized relationships between the variables were tested using moderation and serial mediation analyses.

Findings - Trust between personnel at different hierarchical levels was found to positively influence organizational effectiveness in terms of higher shared awareness of tasks and responsibilities, better information sharing, and, in turn, better decision making. A perceived flat organizational structure and decentralized processes were found to increase flexibility, an increase that in turn improved decision making. Moderation analyses further suggested that trust between hierarchical levels could attenuate the negative effects of personnel's perception of their organization as hierarchical and centralized.

Practical implications - The study results suggest that, at least in Norwegian military contexts, practitioners should be concerned with building trust between personnel at different hierarchical levels, flattening the organizational structure, and decentralizing processes to increase organizational flexibility and effectiveness.

Originality/value - The present study contributes to a better understanding of the role of trust between personnel at different hierarchical levels in the effort to achieve effective organizational structures and processes in military contexts. 
Keywords - Trust, organizational effectiveness, organizational structure, organizational processes.

Article classification - Research paper.

\section{Introduction}

Military and civilian organizations are facing an increasingly wide spectrum of challenges combined with high demands for organizational effectiveness (e.g., Hartnell et al., 2019; Alberts, 2011; Alberts and Hayes, 2003; Bjørnstad, 2013; STO-TR-SAS-085, 2014). As the wealth of information and the complexity of the organizational contexts increase, the sharing of information and the awareness and understanding of tasks and responsibilities in organizations are increasingly essential for the ability to make appropriate decisions that can help organizations achieve their goals. Organizational effectiveness can be understood as key organizational processes, such as shared awareness of tasks and responsibilities, information sharing, and decision making (e.g., Kozlowski and Ilgen, 2005). These key organizational processes have in turn been linked to organizational output (e.g., Benbasat and Lim, 1993; Mesmer-Magnus and DeChurch, 2009; Riley et al., 2006).

Understanding the contingencies for organizational effectiveness is especially urgent in organizations that plan to or are in the process of implementing organizational changes. This has been the case for many military organizations in the wake of the theories of network organization (e.g., Alberts and Hayes, 2003; Bjørnstad, 2011, 2013) and agility (e.g., Alberts, 2011; STO-TRSAS-085, 2014) in military conceptual work. 
Research in international military contexts has suggested that flat structure, decentralized processes, and a high degree of alignment between structure and processes may be beneficial for flexibility and organizational effectiveness at both higher and lower levels of command (Bjørnstad, 2011; Bjørnstad and Lichachz, 2013). Structure is understood here as the formal hierarchical structure of the organization, and processes describe how the structure is implemented in terms of collaborative and decision-making processes (DeSanctis and Poole, 1997). Flexibility is understood as the ability of the organization to respond successfully and adaptively to the complex, unpredictable and changing demands of the environment (Hatum and Pettigrew, 2006). Alignment is understood as the congruence between the organization's structure and processes (Bjørnstad, 2011). Based on the research by Bjørnstad (2011) and Bjørnstad and Lichacz (2013), we thus hypothesize the following:

H1. A flat structure influences organizational effectiveness positively, partly mediated by flexibility.

H2. Decentralized processes influence organizational effectiveness positively, partly mediated by flexibility.

H3. Alignment between flat structure and decentralized processes influences organizational effectiveness positively.

However, the successful delegation of authority to lower hierarchical levels and the decentralization of organizational processes in military and other organizations may depend on other critical issues. Hierarchical levels in military organizations are often very separate, both geographically and in terms of work processes. This suggests that the relationships between people at different hierarchical levels are especially vulnerable and thus especially important to understand. Military theories of network organization (e.g., Alberts and Hayes, 2003) suggest 
that trust is essential for effective network organization (i.e., flat, decentralized, and flexible organization). We understand trust as "a psychological state comprising the intention to accept vulnerability based upon positive expectations of the intentions or behavior of another" (Rousseau et al., 1998, p. 395), a cross-disciplinary definition of trust that is recognized as including the most essential elements of trust (e.g., Burke et al., 2007; De Jong, et al., 2016; Dirks and Ferrin, 2002). Although research has found that trust affects key organizational processes and outcomes (e.g., Bjørnstad et al., 2013; Breuer et al., 2016; Colquitt et al., 2007; De Jong et al., 2016; Dirks and Ferrin, 2002), there is a lack of empirical research on the assumption from network theories that trust is necessary for a flat and decentralized organization to improve effectiveness. However, Bloom et al. (2009) linked trust to decentralization, and a meta-analysis by De Jong et al. (2016) demonstrated that trust between individuals at different hierarchical levels was especially important for team output, suggesting that trust may both interact with organizational variables and influence effectiveness. Based on the above theory and research on trust, we thus propose the following:

H4. Trust between different levels in the organizational hierarchy influences organizational effectiveness positively.

H5. Trust between levels in the organizational hierarchy strengthens (i.e., moderates) the proposed relationships between flat structure and flexibility and flat structure and organizational effectiveness (H1), as well as the proposed relationships between decentralized processes and flexibility and decentralized processes and organizational effectiveness (H2).

As indicated above, organizational effectiveness is understood as shared awareness of tasks and responsibilities, information sharing, and decision making. Research has suggested that these effectiveness indicators may also be internally related in terms of shared awareness of tasks and 
responsibilities and information sharing preceding decision making (e.g., Bjørnstad, 2011;

Bjørnstad and Elstad, 2015; Dennis, 1996; Prince and Salas, 1997; Riley et al., 2006; Yanakiev and Horton, 2012). Based on this research, we anticipate the following:

H6. Shared awareness of tasks and responsibilities and information sharing partly mediate the effects of the independent variables on decision making.

The conceptual model (Figure 1) visualizes the hypotheses and shows how the different variables are anticipated to influence organizational effectiveness in a serial parallel moderated mediation model.

[Insert Figure 1 about here]

\section{Study aims}

The main objective of the present study is to help military and other organizations to increase their organizational effectiveness by improving the understanding of trust between personnel at different hierarchical levels, especially its role in relation to organizational structure and processes. This study puts theoretical assumptions to the test, responds to calls for more integrative organizational theories (e.g., Salas et al., 2017), and focuses on the interdependence of organizational variables, accentuated in a recent meta-analysis (Hartnell et al., 2019).

\section{Method}

\section{Participants and procedures}

Survey data were collected from a Norwegian national military exercise organization at operational/headquarter (HQ, i.e., higher hierarchical level) and tactical (i.e., lower hierarchical level) levels of command in 2015. The purpose of the exercise was to train personnel in a scenario escalating from a national crisis to a NATO Article 5 conflict (i.e., full armed conflict entailing the collective defense of all NATO members), including both operational and tactical 
levels of command. The exercise was a command post exercise (CPX, i.e., run by an exercise command). There was collaboration both between the hierarchical levels (HQ and tactical) and between the nine different tactical units that participated in the exercise. The collaboration between the two different hierarchical levels and the different tactical units was primarily technologically mediated (i.e., computer based). The current research focuses on the relationship between the two hierarchical levels, the operational level (i.e., HQ) and the tactical level of command.

Questionnaires were distributed electronically and completed towards the end of the exercise (i.e., at one time) by all participants. All participants answered all measures/questions, were informed of the purpose of the study, and participated on a free-will basis. The survey was scrutinized and accepted by an HQ organizational development team, the exercise lead, and the highest-ranking commander. All information obtained was anonymized and treated with confidentiality.

There was a total of 171 participants after correcting for missing values on the studied variables. The response rate was $43 \%$ (44\% at the tactical level and $42 \%$ at the operational level of command). The sample demographics are presented in Table I.

[Insert Table I about here]

\section{Measures}

Flat structure, decentralized processes, flexibility, alignment, trust, and the organizational effectiveness variables (i.e., shared awareness of tasks and responsibilities, information sharing, and decision making) were measured using scales developed for use in military contexts and whose psychometric properties were tested in Bjørnstad and Elstad (2015). These measures were based on earlier work by Bjørnstad et al. (2013), Lichacz and Bjørnstad (2013), Bjørnstad (2005, 
2011), and Yanakiev and Horton (2012). Because all participants were Norwegian, all measures were in Norwegian.

Flat structure, decentralized processes and flexibility were measured using Bjørnstad's measures (Bjørnstad, 2015; Bjørnstad and Elstad, 2015), which were expansions of Bjørnstad's single-item measures (2011). Each of these constructs was measured with a unique five-item, five-point scale. Flat structure had one item answered on a bipolar scale from "very hierarchical" (1) to "very flat" (5) and four items answered on a Likert scale from "strongly disagree" (1) to "strongly agree" (5). The lead stem of the four Likert scale items was "Indicate to what degree you agree or disagree with the following statements", and a sample item was "There are few decision-making levels in this exercise organization”. High scores indicate a flat structure. Cronbach's alpha (based on standardized items) demonstrated acceptable reliability of the measure $(\alpha=.71)$. Decentralized processes were measured using a bipolar scale with the response choices "very centralized" (1) to "very decentralized" (5). The lead stem was "From your standpoint in this exercise, how would you describe the following organizational processes in terms of centralization/decentralization?", and a sample item was "Work processes". High scores indicate decentralized processes. The measure demonstrated acceptable reliability $(\alpha=$ .71). Flexibility was measured using a bipolar scale with the response choices "very rigid" (1) to "very flexible" (5). The lead stem was "From your standpoint in this exercise, how would you describe the following organizational processes in terms of rigidity/flexibility?", and a sample item was "Distribution of responsibilities". High scores indicate flexibility. The reliability of the measure was $\operatorname{good}(\alpha=.81)$.

Alignment was an estimate of the level of congruence between structure and processes (Bjørnstad, 2011); it was calculated as the difference between the structure and processes scores. 
Hence, the value 5 on both structure and processes gives an alignment score of 0 , and the values 1 and 5 on structure and processes yield an alignment score of -4 . The close-to-zero mean value, $M=-.64$ (Table II), indicates a high level of congruence between the structure and process values. Hence, scores close to zero indicate a high degree of alignment between structure and processes.

Trust was measured using the metric from Bjørnstad et al. (2013; Bjørnstad, 2013). This is a task-focused cognitive trust measure that was developed and validated in military contexts, hence fitting our focus and goal. The metric has three items answered on a five-point bipolar scale, with answer categories from "very confident" (1) to "very doubtful" (5), recoded so that high scores indicate high trust. The metric was adapted to measure trust between the two levels of command. The lead stem was "During this exercise, how confident have you been that:", and a sample item was "Your colleagues at the operational/tactical level of command fulfill their responsibilities?" The measure demonstrated good reliability $(\alpha=.85)$. Hence, personnel from the tactical level of command (i.e., subordinate level) answered pertaining to their colleagues at the operational level of command (i.e., superior level) and vice versa. This means that each level of command rated their trust in the other level of command.

Organizational effectiveness was measured by the three constructs shared awareness of tasks and responsibilities, information sharing, and decision making. The information-sharing and decision-making measures were a refinement of Bjørnstad's original measures (2011), and the shared awareness of tasks and responsibilities measure was developed on the basis of four items from Yanakiev and Horton's (2012) measure. All three measures were validated in Bjørnstad and Elstad (2015). 
Shared awareness of tasks and responsibilities was measured by four items answered on a five-point Likert scale with answer categories from "strongly disagree" (1) to "strongly agree" (5). The metric was adapted to measure shared awareness of tasks and responsibilities between the tactical and operational levels of command; hence, the questions were focused on the other hierarchical level. The lead stem was "Indicate to what degree you agree or disagree with the following statements based on the role that you have in this exercise:". A sample item (item 2) was "The HQ and the tactical commands/our tactical command" are aware of each other's areas of responsibility" (* indicates that the phrasing was adapted to the respondents' level of command: the operational level related to "tactical commands" in general, while the tactical level related to their own tactical command unit). High scores indicate a high degree of shared awareness of tasks and responsibilities; items 1, 3 and 4 were thus recoded. The measure demonstrated good reliability $(\alpha=.85)$.

Information sharing was measured by three items answered on a 5-point scale from "very content" (1) to "very discontent" (5). It was recoded so that high scores indicate good information sharing. The lead stem was "Indicate which answer best describes your perception based on the role that you have in this exercise:", and a sample item was "How content are you with the content of the information that you receive?". The measure demonstrated acceptable reliability $(\alpha=.73)$.

Decision making was measured by three items. The lead stem was "Indicate which answer best describes your perception based on the role that you have in this exercise:", and a sample item (item 2) was "How do you perceive the decision quality?" The corresponding response choices ranged from "very good" (1) to "very poor" (5). Items 2 and 3 were recoded so that high scores indicate good decision making. The measure demonstrated acceptable reliability ( $\alpha=.73$ ). 
Covariates. We controlled for the demographic variables age, gender, level of command, civilian educational level, military educational level, military services, rank, and years in military service in the analyses. These variables were not found to influence the results and are therefore not presented in the subsequent results section.

Statistical analyses

For participants who had one value missing on a measure, the value was replaced by the average score of the other items measuring the same construct. A total of six missing values were therefore replaced in the data sets of six different respondents. Data from participants who had more missing values were excluded from the analyses (6 respondents).

The hypothesized relationships between the variables (Figure 1) were analyzed using the PROCESS function V3.4 in IBM SPSS V.26 (Hayes, 2018). The analyses were performed in two main steps. First, PROCESS model 81 was used to estimate serial parallel mediation effects.

Second, PROCESS model 8 was used to test for both moderation effects and moderated mediation effects of trust. The variables not involved in the moderation effects were included as covariates in the analyses using model 8 . Nonparametric analyses using 5000 bootstrapped samples were applied to estimate $95 \%$ confidence intervals for indirect effects and moderated mediation effects. A multiple-group structural equation model was used to test the cross-validity of the mediation model, i.e. whether the regression coefficients were equal in the sample at the tactical level (i.e., subordinate level) and in the sample at the operational level (i.e., superior level) (see e.g. Hayes, 2018; Tabachnick \& Fidell, 2013). IBM SPSS AMOS 25.0 was applied for this purpose.

\section{Results}


Descriptive statistics and zero-order correlations are presented in Table II. The assumptions of linearity and univariate and multivariate normality necessary for more advanced analyses were evaluated. Trust was found to be marginally acceptable in terms of normality, with a somewhat high kurtosis value (1.25); all other measures had acceptable values.

[Insert Table II about here]

\section{Serial parallel mediation effects}

The estimated direct effects between the variables in the serial parallel mediation model using PROCESS model 81 are presented in Figure 2. The estimated indirect effects from model 81 are presented in Table III.

[Insert Figure 2 about here]

[Insert Table III about here]

Figure 2 suggests that flat organizational structure influences organizational effectiveness in terms of improved decision making, both directly and indirectly, through flexibility. These results indicate partial support for hypothesis 1 (partial because flat structure was significantly related to only one of the three measures of organizational effectiveness). The estimated model further indicated that the effects of decentralized processes on decision making were fully mediated by flexibility, meaning that decentralized processes increased flexibility, an increase that in turn improved decision making. This finding partially supported hypothesis 2 (partially because no direct effects of decentralized processes on the organizational effectiveness measures were found). Alignment was not found to be significantly related to the organizational effectiveness measures; hypothesis 3 was thus not corroborated. The analyses depicted in Figure 2 further lend support to hypothesis 4; trust had a direct positive effect on shared awareness of tasks and responsibilities and information sharing. Trust was found to have indirect effects on 
decision making through shared awareness and information sharing, thus supporting hypothesis 6. Hypothesis 6 was, however, only partly supported because shared awareness of tasks and responsibilities and information sharing were not found to mediate the effects of flat structure and decentralized processes on decision making. The validity of the mediation model (Figure 2) across the two organizational hierarchical levels was tested by comparing the fit of a constrained model (i.e., all nine path coefficients were set to be equal in the two sub-samples) with an unconstrained model (i.e., all nine path coefficients values could vary freely within the two subsamples). The results showed no significant difference between the two models $\left[\chi^{2}\right.$ diff $(9)=9.85$, $p=.36$, suggesting generalizability of the model to both the tactical and the operational level of command.

To examine hypothesis 5, the four hypothesized moderating effects of trust (Figure 1) were tested in two separate analyses using PROCESS model 8 . The first analysis found that the inclusion of the product term between trust and flat structure gave a significant increase in the amount of explained variance in decision making $\left(\Delta R^{2}=.04, p<.01\right)$, denoting a significant moderating effect of trust on the direct effect of flat structure on decision making. A visual representation of the moderating effect of trust is presented in Figure 3. A subsequent simple slope analysis demonstrated that the relationship between organizational structure and decision making was statistically significant when trust was low $(-1 \mathrm{SD}, b=0.49, p<.001)$ and average $(b=0.28, p<.001)$ but not when trust was high $(+1 \mathrm{SD}, b=0.07, p=.44)$. This means that a hierarchical structure depends more on trust to be efficient compared to a flat structure, which is the opposite of what was anticipated in hypothesis 5. Trust was not found to moderate the relationship between flat structure and the mediator flexibility, as proposed in hypothesis 5). 
The second analysis using PROCESS model 8 found that the inclusion of the product term between trust and decentralized processes gave a significant increase in the amount of explained variance in flexibility $\left(\Delta R^{2}=.02, p<.05\right)$, indicating that the effect of decentralized processes on flexibility was moderated by trust. A visual representation of the moderating effect of trust is displayed in Figure 3. This means that an organization with centralized processes depends more on trust to be flexible - the opposite of what was anticipated in hypothesis 5 . Simple slope analysis found that the effect of decentralized processes was statistically significant at all levels of trust, at low trust $(-1 \mathrm{SD}, b=0.57, p<.001)$, at average trust $(b=0.40, p<.001)$, and at high trust $(+1 \mathrm{SD}, b=0.24, p<.05)$. Trust was not found to moderate the direct effect between decentralized processes and decision making (also proposed in hypothesis 5).

[Insert Figure 3 about here]

The moderating effect of trust also suggests that the mediating role of flexibility between decentralized processes and decision making is contingent upon the level of trust, indicating a moderated mediation effect. Table IV shows that the indirect effect of decentralized processes on decision making is significant at low and medium levels of trust but that the effect becomes nonsignificant and close to zero at high levels of trust. This means that decentralized processes are especially important for flexibility and the quality of decision making when trust is low between the different organizational hierarchical levels.

[Insert Table IV about here]

\section{Discussion}

The purpose of the present study was to test theoretical assumptions to support military and other organizations in their work towards increased organizational effectiveness by improving 
the understanding of the effects of trust between different hierarchical levels, including its role in relation to different organizational structures and processes.

The results supported the suggestions ( $H 1$ and $H 2)$ that flat structure and decentralized processes have positive effects on both flexibility and organizational effectiveness (effectiveness as measured by decision making). The effect of decentralized processes on decision making was fully mediated by flexibility, while the effects of flat structure on decision making were partly mediated by flexibility.

The results did not support the proposition (H3) that alignment between structure and processes has direct effects on organizational effectiveness in terms of decision making.

The effectiveness measures were also found to be interrelated; shared awareness of tasks and responsibilities and information sharing both related positively to decision making (H6).However, shared awareness of tasks and responsibilities and information sharing were not found to mediate the effects of flat structure and decentralized processes on decision making. Hence, H6 was only partly supported. In line with the literature (e.g., Dennis, 1996; Bjørnstad, 2011) and based on the understanding that shared awareness and information sharing are necessary but not sufficient conditions for good decision making and not vice versa, these results suggest that shared awareness of tasks and responsibilities and information sharing both influenced decision making.

The results lend support to the suggestion $(H 4)$ that trust between hierarchical levels influences organizational effectiveness positively in terms of higher shared awareness of tasks and responsibilities, better information sharing, and, in turn, better decision making. The suggestion (H5) that trust strengthens the effects of flat structure, decentralized processes, and flexibility on organizational effectiveness was not supported, although two moderating effects of trust was found. Both of the moderating effects of trust gave a substantial increase in explained 
variance, but were in the opposite direction of what was expected. The first effect indicated that a hierarchical structure decreases the quality of decision making primarily at low levels of trust, but not when trust was high. A flat structure was not dependent upon trust to increase the quality of decision making. The second moderating effect of trust showed that centralized processes decrease the quality of decision making through reducing flexibility at low and medium levels of trust, but not when trust was high. Hence, high levels of trust may be understood as restricting the negative effects of centralized processes and hierarchical structure on flexibility and decision making.

\section{Theoretical and practical implications}

The current results lend support to the findings in Bjørnstad (2011) and Bjørnstad and Lichacz (2013) by demonstrating positive direct and indirect effects of flat structure on organizational effectiveness, positive indirect effects of decentralization on organizational effectiveness, positive direct effects of flexibility on organizational effectiveness, and a positive relationship between the effectiveness variables, information sharing and decision making.

The current study expands the understanding of the antecedents of organizational effectiveness by incorporating trust into the organizational effectiveness model of Bjørnstad (2011), thereby integrating different lines of research, in line with Salas et al.'s (2017) call for more integrative organizational research. The final moderated mediation model adds to our knowledge of organizational interdependencies relevant for both theory and practice - a factor also underlined in Hartnell et al.’s (2019) meta-analysis.

The results also provide empirical evidence in support of central theories in NATO conceptual research - the theories of network organization (e.g., Alberts and Hayes, 2003) and 
the agility concept (e.g., STO-TR-SAS-085, 2014). These theories have emphasized decentralization (often called "power to the edge"), flatter hierarchies, and trust as important factors in flexible and effective military organizations. However, the present research raises doubt as to the necessity of trust for flat and decentralized types of organizations to be effective. Rather, the results suggest that hierarchical and centralized organizations may be more dependent on trust between personnel at different hierarchical levels and that flat and decentralized types of organizations are more robust (in terms of being less dependent upon trust).

The current research also lends support to the centrality of information sharing and decision making purported in the military conceptual theories referred to above. Information exchange seems pivotal from both a conceptual military standpoint and from an organizational development standpoint.

The results of this research may help military and other organizations by highlighting the essential role of trust in addition to flat structure and decentralized processes for organizational effectiveness. Appreciating the centrality of trust between personnel at different hierarchical levels for organizational effectiveness may increase the probability of organizations investing the necessary time to build trust between their members at different hierarchical levels.

\section{Limitations and future directions}

The analyses were based on cross-sectional data, making it important to pay attention to issues concerning endogeneity. Although the results provide support for the suggested causal relationships between the variables in the model, the model does not provide proof of such causal relationships. The direction of the causal relationships between the constructs was therefore evaluated on a thorough theoretical basis (e.g., Bjørnstad, 2011; Bjørnstad, et al., 2013; Colquitt, 
et al., 2007; De Jong et al., 2016; Dennis, 1996; Hirschfeld et al., 2006; Mathieu et al., 2015; Riley, et al., 2006; Valaker et al., 2016).

The use of self-report measures may be seen as a limitation because of the common method bias (e.g., Campbell \& Fiske, 1959; Cronbach \& Meehl, 1955; Doty \& Glick, 1998), potentially also causing endogenity. However, both Doty and Glick (1998) and Conway and Lance (2010) argue that the problem of common method bias is substantially exaggerated in organizational research. Indeed, the work of Conway and Lance (2010) indicates that the problem of underestimating relationships when using different methods (Type II error) is greater than that of overestimating relationships when using the same methods (Type I error). Doty and Glick (1998) suggest that employing different wordings and different response formats in self-report measures will serve to minimize the common method bias. Therefore, to minimize this bias, the response formats employed to measure the different variables were deliberately distinct for each variable, employing bipolar descriptive measurement scales, in addition to one Likert and one combined Likert and bipolar descriptive scale. Additionally, some of the scales were reverse coded (i.e., trust, information sharing, and parts of the decision-making and shared awareness measures). The use of different bipolar descriptive scales instead of the more common Likert-type scales with identical response denominators (agree/disagree), and also reverse coding, should thus lessen the risk of common method bias, hence also the risk of endogeneity.

In line with the research on which this study was based (e.g., Bjørnstad, 2011; McAllister, 1995; Valaker et al., 2016), the results of this study are based on participants' perceptions and not on direct organizational measures. Subjective experiences such as trust and shared awareness of tasks and responsibilities also naturally lend themselves to the use of subjective measures. Moreover, the use of participants' subjective perceptions to make inferences about an 
organization's characteristics is often considered the most relevant approach in organizational studies (Patterson et al., 2005; Spector, 1994). An organization may be understood in terms of the sum of the realities perceived by its participants (e.g., Patterson et al., 2005). Hence, this research is not a study of different objective realities in different organizations but rather reflects the different perceptions of many individuals pertaining to their different working environments in one organization. The current response rate of $43 \%$ may be considered low. However, because our goal was to investigate the relations among the variables rather than estimate population values, this is not considered a serious flaw (Shadish et al., 2002). The response rates for the two hierarchical levels in the organization were also quite similar ( $42 \%$ at the operational/HQ level and $44 \%$ at the tactical level), excluding this factor from being the cause of any systematic error. Additionally, the relationships between the variables in the model were found to be equal at the tactical and the HQ levels, demonstrating robustness of the proposed model.

To test the strength of the current results, it may also be useful to employ experimental manipulations and direct objective measures. Selected parts of the model may also be studied more in depth across contexts, like whether and how the role of flexibility may be different or the same in military and business types of contexts. Future research should also test the whole model in other types of organizations, both military and civilian, in different national contexts, and at different hierarchical levels to assess the generalizability of the results.

It may also be beneficial to assess the conceptual model in larger samples because larger samples have more power to detect significant effects, which is especially important for the proposed moderating effects. To assess the generalizability of results across organizations and cultures, in effect also involving larger samples, we suggest larger research ventures with data collection from many different types of organizations in different countries and cultures. This 
would allow for conducting multilevel analysis (MLM), with organization type and culture at level II and III respectively. In such a research venture, the type of organization and culture would be controlled for, as well as allowing for any interaction effects with the organizational level to be revealed.

A suggestion for future research could also be to look more closely at trust. The current research focused on trust between two different organizational hierarchical levels, because research has been lacking here. However, it may also be of value to understand how trust between two different organizational hierarchical levels compares to trust within each hierarchical level, as well as with trust within and across different military services (army, navy, etc). Finally, we would like to promote the expansion of the model to include additional variables vital to organizational effectiveness.

\section{Conclusion}

Contrary to expectations, our data suggested that flatter and more decentralized organizations may be more flexible and effective irrespective of trust and that negative effects of a hierarchical and centralized organization can be attenuated by trust between levels in the organizational hierarchy. This means both that trust may be seen as a powerful tool and that flat and decentralized organizations may be seen as the more robust organizational form - at least in Norwegian military contexts. The findings may provide an extra impetus for an increased focus on issues central to the building of good relations between organizational hierarchical levels. 


\section{References}

Alberts, D.S. (2011), The agility advantage: A survival guide for complex enterprises and endeavors, DoD CCRP Publication series, Washington, DC.

Alberts, D.S. and Hayes, R.E. (2003), Power to the edge: Command and control in the information age, DoD CCRP Publication series, Washington, DC.

Benbasat, I. and Lim, L.-H. (1993), "The effects of group, task, context, and technology on the usefulness of group support systems: A meta-analysis of experimental studies", Small group research, Vol. 24 No. 4, pp. 430-462.

Bjørnstad, A.L. (2005), Part I: Allied Warrior 2004 - Pilot study and analysis of cross-cultural organizational issues, FFI report 2005/01709, Norwegian Defence Research Establishment (FFI), Kjeller, Norway.

Bjørnstad, A.L. (2011), "Exploring network organization in military contexts - effects of flatter structure and more decentralized processes”, Military Psychology, Vol. 23 No. 3, pp. 315331.

Bjørnstad, A. L. (2015). “Scales for measuring three key organizational constructs”, paper presented at the $17^{\text {th }}$ Congress of the European Association of Work and Organizational Psychology (EAWOP): Respectful and effective leadership - managing people and organizations in turbulent times, 20-23 May, Oslo, Norway, available at: http://eaw.pg2.at/abstracts/a1356.html?zoom highlight= (accessed 18 September, 2017).

Bjørnstad, A.L. (2013), Network organization pitfalls and success factors for team and organizational processes: Analyses of Key Organizational Variables and Cultural Differences in International Military Contexts, doctoral dissertation, Department of Psychology, Faculty of Social Sciences, University of Oslo, Norway, available at: 
https://www.duo.uio.no/handle/10852/37945.

Bjørnstad, A.L. and Elstad, A.K. (2015), Utvikling og evaluering av spørreskjema med fokus på organisasjon og bruk av samhandlingsteknologi, FFI report 2015/00046, Norwegian Defence Research Establishment (FFI), Kjeller, Norway.

Bjørnstad, A.L., Fostervold, K.I. and Ulleberg, P. (2013), "Effects of cultural diversity on trust and its consequences for team processes and outcomes in ad hoc distributed teams", Scandinavian Journal of Organizational Psychology, Vol. 5 No. 1, pp. 3-15.

Bjørnstad, A.L. and Lichacz, F.M.J. (2013), “Organizational flexibility from a network organizational perspective: A study of central predictors and moderating factors in military contexts", Leadership and Organizational Development Journal, Vol. 34 No. 8, pp. 763-783.

Bloom, N., Sadum, R. and Van Reenen J. (2009), "The organization of firms across countries", MA: NBER Working paper No. 151129, Cambridge, available at: http://www.nber.org/papers/w15129.

Breuer, C., Hüffmeier, J. and Hertel, G. (2016), "Does trust matter in virtual teams? A metaanalysis of trust and team effectiveness considering virtuality and documentation as moderators", Journal of Applied Psychology, Vol. 101 No. 8, pp. 1151-1177.

Burke, C.S., Sims, D.E., Lazarra, E.H. and Salas, E. (2007), “Trust in leadership: A multi-level review and integration", The leadership quarterly, Vol. 18, pp. 606-632.

Campbell, D. T., \& Fiske, D. W. (1959), "Convergent and discriminant validation by the multitrait-multimethod matrix", Psychological bulletin, Vol. 56, pp. 81-105.

Conway, J. M., \& Lance, C. E. (2010), “What reviewers should expect from authors regarding common method bias in organizational research", Journal of business psychology, Vol. 25, pp. 325-334. 
Cronbach, L. J., \& Meehl, P. E. (1955), "Construct validity in psychological tests", Psychological bulletin, Vol. 52, pp. 281-302.

Doty, D. H, \& Glick, W. H. (1998), "Common method bis: does common methods variance really bias results?", Organizational research methods, Vol. 1 No.4, pp. 374-406.

Colquitt, J.A., Scott, B.A. and LePine, J.A. (2007), “Trust, trustworthiness, and trust propensity: A meta-analytic test of their unique relationships with risk taking and job performance", Journal of applied psychology, Vol. 92 No. 4, pp. 909-927.

De Jong, B.A., Dirks, K.T. and Gillespie, N. (2016), “Trust and Team Performance: A MetaAnalysis of Main Effects, Moderators, and Covariates”, Journal of Applied Psychology, Vol. 101 No. 8, pp. 1134-1150.

DeSanctis, G. and Poole, M.S. (1997), "Transitions in teamwork in new organizational forms", Advances in group processes, Vol. 14, pp. 157-176.

Dirks, K.T. and Ferrin, D.L. (2002), “Trust in leadership: Meta-analytic findings and implications for research and practice", Journal of applied psychology, Vol. 87 No. 4, pp. 611-628.

Dennis, A.R. (1996), “Information exchange and use in group decision making: you can lead a group of information, but you can't make it think", MIS Quarterly, Vol. 20 No.4, pp.433457.

Hartnell, C.A., Ou, A.Y., Kinicki, A.J., Choi, D. and Karam, E.P. (2019), “A Meta-Analytic Test of Organizational Culture's Association With Elements of an Organization's System and Its Relative Predictive Validity on Organizational Outcomes”, Journal of Applied Psychology, Vol. 104 No. 6, pp. 832-850.

Hatum, A. and Pettigrew, A.M. (2006), "Determinants of organizational flexibility: a study in an 
emerging economy", British journal of management, Vol. 17, pp. 115-137.

Hayes, A. F. (2018), Introduction to Mediation, Moderation and Conditional Process Analysis. A Regression-Based Approach ( ${ }^{\text {nd }}$ ed), the Guilford Press, New York.

Hirschfeld, R.R., Jordan, M.H., Feild, H.S., Giles, W.F. and Armenakis A.A. (2006), “Becoming team players: Team members'mastery of teamwork knowledge as a predictor of team task proficiency and observed teamwork effectiveness", Journal of Applied Psychology, Vol. 91 no. 2 , pp. $467-474$.

Kozlowski, W.J. and Ilgen, D.R. (2005), "Enhancing the effectiveness of work groups and teams" Psychological science in the public interest, Vol. 7 No. 3, pp. 77-124.

Lichacz, F.M.J. and Bjørnstad, A.L. (2013), “Are linguistic differences in multinational coalitions as problematic as we think? No, not really", Military Psychology, Vol. 25 No. 1, pp. 57-69.

McAllister, D.J. (1995), "Affect- and cognition-based trust as foundations for interpersonal cooperation in organizations", Academy of management journal, Vol. 38 No. 1, pp. 24-59.

Mesmer-Magnus, J.R. and DeChurch, L.A. (2009), “Information and team performance: A metaanalysis", Journal of applied psychology, Vol. 94 No. 2, pp. 535-546.

Patterson, M.G., West, M.A., Shackleton, V.J., Dawson, J.F., Lawthom, R., Maitlis, S., Robinson, D.L. and Wallace, A.M. (2005), "Validating the organizational climate measure: links to managerial practices, productivity and innovation", Journal of organizational behavior, Vol. 26, pp. 379-408.

Prince, C. and Salas, E. (1997), "Situation assessment for routine flight and decision making", International Journal of Cognitive Ergonomics, Vol. 1 No. 4, pp. 315-324. 
Riley, J.M., Endsley, M.R., Bolstad, C.A. and Cuevas, H.M. (2006), “Collaborative planning and situation awareness in army command and control", Ergonomics, Vol. 49, pp. 1139-1153.

Rousseau, D.M., Sitkin, S.B., Burt, R.S. and Camerer, C. (1998), "Not so different after all: A cross-discipline view of trust", Academy of management review, Vol. 23 No. 3, pp. 393404.

Salas, E., Kozlowski, S.W. J. and Chen, G. (2017), “A century of progress in industrial and organizational psychology: Discoveries in the next century", Journal of Applied Psychology, Vol. 102 No. 3, pp. 589-598.

Shadish, W.R., Cook, T.D. and Campbell, D.T. (2002), Experimental and quasi-experimental designs for generalized causal inference, Houghton Mifflin Company, Boston.

Spector, P.E. (1994), "Using self-report questionnaires in OB research: a comment on the use of a controversial method", Journal of organizational behavior, Vol. 15, pp. 385-392.

STO-TR-SAS-085 (2014), Command and control (C2) Agility: Task Group SAS-085 final report, NATO STO Publisher (ISBN 978-92-837-0206-1).

Tabachnick, B. G., and Fidell, L. S. (2013), Using multivariate statistics (6 ${ }^{\text {th }}$ ed), Pearson Education, Inc., Boston.

Yanakiev. Y. and Horton. J.S. (Eds.) (2012), Improving the Organisational Effectiveness of Coalition Operations: Task Group HFM-163 final report, NATO STO Publisher (ISBN: 978-92-837-0175-0). 
Table I. Participant demographics

\begin{tabular}{|c|c|c|c|c|c|c|}
\hline & $n$ & $\%^{*}$ & Mean / SD & & $n$ & $\%^{*}$ \\
\hline Age (Range: 21 - 59) & & & $45.7 / 8.8$ & Highest completed civilian education/degree: & & \\
\hline Gender: & & & & High school & 70 & 40.9 \\
\hline Male & 163 & 95.3 & & Bachelor's degree (civilian) & 60 & 35.1 \\
\hline Female & 7 & 4.1 & & Master's degree (civilian) & 29 & 17.0 \\
\hline Military & 167 & 97.7 & & Ph.D. (civilian) & 0 & 0 \\
\hline Years of military service (Range: $1-39$ ) & & & $24.0 / 10.1$ & Other (civilian) & 12 & 7.0 \\
\hline Civilian & 4 & 2.3 & & Highest completed military education/degree: & & \\
\hline Level of command: & & & & Officer candidate school & 21 & 12.3 \\
\hline HQ level & 94 & 55 & & Bachelor's degree (military) & 96 & 56.1 \\
\hline Tactical level & 77 & 45 & & Master's degree (military) & 32 & 18.7 \\
\hline Services: & & & & Ph.D. (military) & 0 & 0 \\
\hline Army & 62 & 36.6 & & Other (military) & 15 & 8.8 \\
\hline Navy & 38 & 22.2 & & NATO rank: & & \\
\hline Air Force & 45 & 26.3 & & Other ranks: OR4-OR5 & 1 & .6 \\
\hline Home Guard & 20 & 11.7 & & Officers: OF1 & 10 & 5.8 \\
\hline \multirow[t]{3}{*}{ Special Forces } & 1 & .6 & & Officers: OF2-OF3 & 92 & 53.8 \\
\hline & & & & Officers: OF4-OF6 & 61 & 35.7 \\
\hline & & & & Officers: OF7-OF9 & 2 & 1.2 \\
\hline
\end{tabular}

Notes. There was a $43 \%$ response rate. $N$ corrected for missing values on the studied variables $=171 .{ }^{*}$ Calculated from the whole sample. Due to some missing values, the numbers do not add up to $100 \%$ for all demographics. 
Table II. Means, standard deviations, and zero-order correlation coefficients

\begin{tabular}{|c|c|c|c|c|c|c|c|c|c|}
\hline & Mean & $S D$ & 1 & 2 & 3 & 4 & 5 & 6 & 7 \\
\hline 1. Trust $\left(X_{1}\right)$ & 3.62 & .73 & & & & & & & \\
\hline 2. Flat structure $\left(X_{2}\right)$ & 2.72 & .63 & -.013 & & & & & & \\
\hline 3. Decentralized processes $\left(X_{3}\right)$ & 2.69 & .57 & .067 & .062 & & & & & \\
\hline 4. Alignment $\left(X_{4}\right)$ & 0.63 & .51 & -.113 & $.159^{*}$ & -.119 & & & & \\
\hline 5. Flexibility $\left(M_{1}\right)$ & 3.06 & .66 & .057 & $.250^{* *}$ & $.379^{* * *}$ & -.042 & & & \\
\hline 6. Shared awareness $\left(M_{2 a}\right)$ & 3.46 & .71 & $.496^{* * *}$ & .050 & $.164^{*}$ & -.112 & .141 & & \\
\hline 7. Information sharing $\left(M_{2 b}\right)$ & 3.85 & .59 & $.236^{* *}$ & .040 & .077 & -.069 & .126 & $.210^{* *}$ & \\
\hline 8. Decision making $(Y)$ & 3.59 & .69 & $.268^{* * *}$ & $.296^{* * *}$ & $.215^{* *}$ & $-.154^{*}$ & $.364^{* * *}$ & $.429^{* * *}$ & $.289^{* * *}$ \\
\hline
\end{tabular}

Notes. High scores indicate flat structure, decentralized processes, low alignment of structure and processes, high trust, high flexibility, high shared awareness of tasks and responsibilities, good information sharing, and good decision making. All variables are on a scale from 1-5 except alignment, which is a difference score between the mean score for structure and the mean score for processes (i.e., no difference $=0$, maximum difference $=-4$ ). ${ }^{*} p<.05 . * * p<.01 . * * * p<.001 . N=171$. 
Table III. Standardized indirect effects on decision making

\begin{tabular}{lcccccc}
\hline & $\begin{array}{c}\text { Through } \\
\text { flexibility } \\
X i-M_{1}>Y\end{array}$ & $\begin{array}{c}\text { Through } \\
\text { shared } \\
\text { awareness } \\
X_{i->M 2 a}>Y\end{array}$ & $\begin{array}{c}\text { Through info. } \\
\text { sharing } \\
X_{i->}>M_{2 b}>Y\end{array}$ & $\begin{array}{c}\text { Serial } \\
\text { mediation } \\
X i->M 1-> \\
M 2 a->Y\end{array}$ & $\begin{array}{c}\text { Serial } \\
\text { mediation } \\
X_{i-}>M 1-> \\
M 2 b->Y\end{array}$ & $\begin{array}{c}\text { Total indirect } \\
\text { effect }\end{array}$ \\
\hline Trust $\left(X_{1}\right)$ & $.01[-.03, .04]$ & $.15[.07,23]$ & $.04[.01, .09]$ & $.00[-.00, .01]$ & $.00[-.00, .01]$ & $.20[.10, .29]$ \\
Flat structure $\left(X_{2}\right)$ & $.05[.01, .10]$ & $.01[-.03, .07]$ & $.00[-.03, .04]$ & $.01[-.01, .02]$ & $.00[-.01, .02]$ & $.08[.01, .15]$ \\
Decentralized processes $\left(X_{3}\right)$ & $.08[.02, .14]$ & $.03[-.01, .08]$ & $.00[-.04, .04]$ & $.01[-.01, .03]$ & $.01[-.01, .02]$ & $.12[.04, .21]$ \\
\hline
\end{tabular}

Notes. Standardized coefficients with 95\% CI in brackets estimated from bootstrapped standard errors. Bold $=$ significant at the $5 \%$ level. $N=171$. 
Table IV. Moderated mediation effect of decentralized processes on decision making at specific conditional values of trust (unstandardized regression coefficients)

\begin{tabular}{lcc}
\hline Specific conditional values of trust & Indirect effect & $95 \% \mathrm{Cl}$ \\
\hline-1 SD below the mean & $\mathbf{0 . 1 3 8}$ & {$[0.043,0.230]$} \\
Mean & $\mathbf{0 . 0 9 7}$ & {$[0.030,0.174]$} \\
+1 SD above the mean & 0.057 & {$[-0.001,0.146]$} \\
\hline
\end{tabular}

Notes. Decentralized processes and trust are mean centered. Bold $=$ significant at the $5 \%$ level. $N=171$. 
Trust and organization 31

Figures

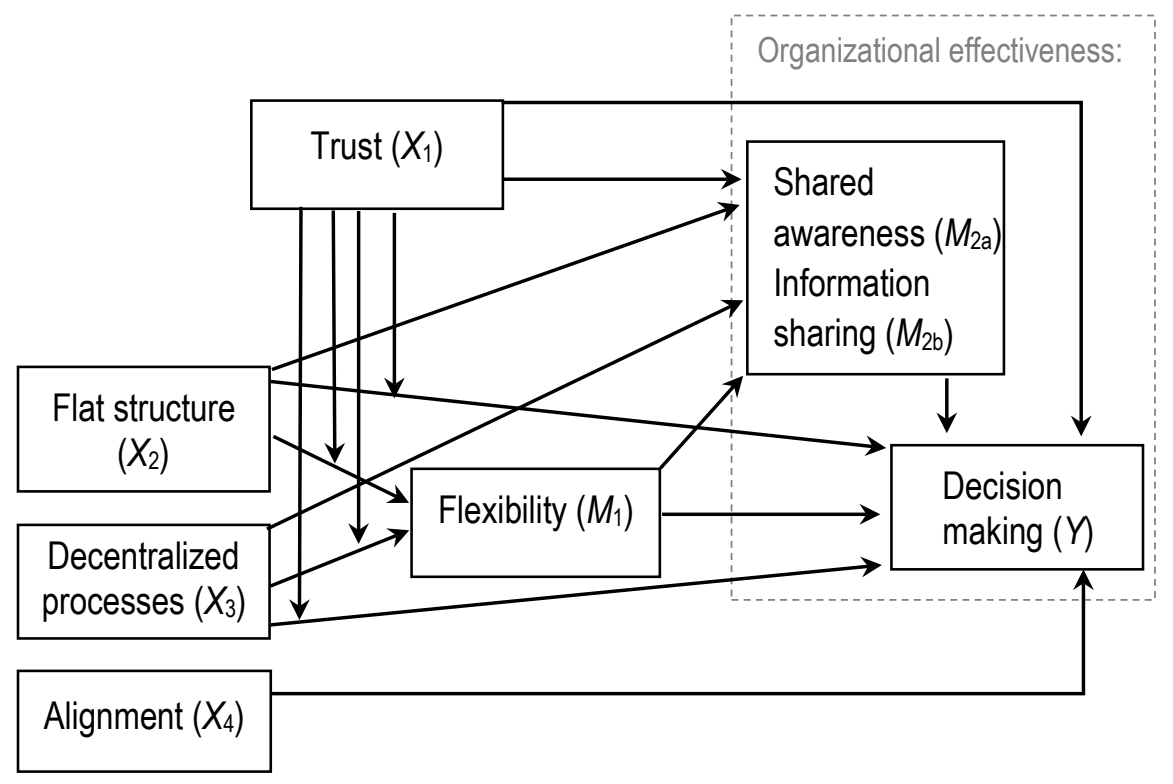

Figure 1. Conceptual model. 


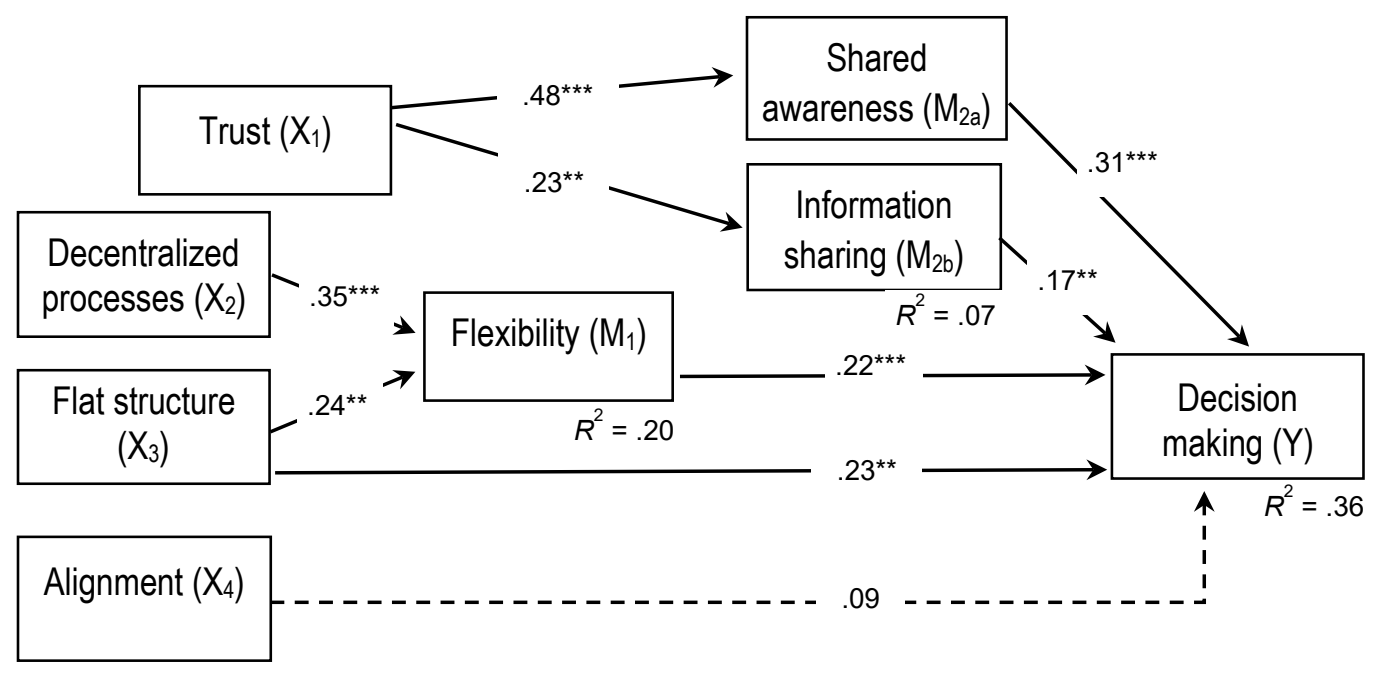

Figure 2. Final mediation model: Hypothesized paths supported in current research.

Standardized coefficients presented. ${ }^{*} p<.05,{ }^{*} p<.01, * * * p<.001 . N=171$. 

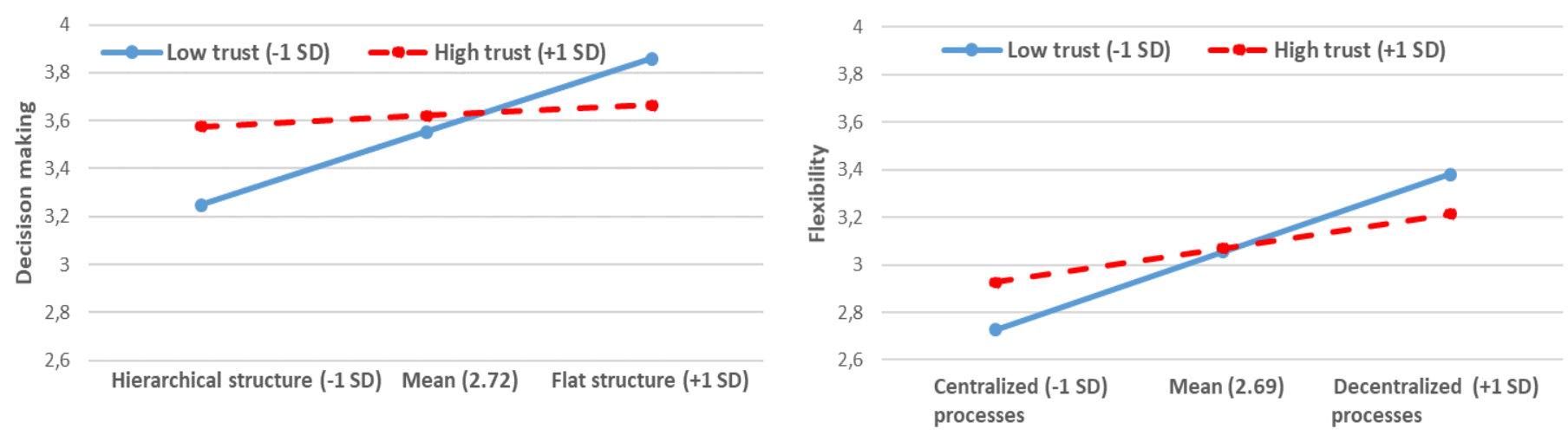

Figure 3. Moderating effects of trust on the relationship between flat structure and decision making (left) and decentralized processes and flexibility (right). The slopes were estimated on the basis of unstandardized regression coefficients $(N=171)$. 